\title{
Erfolge der modernen Wundversorgung
}

\author{
Erster Wundreport zeigt weniger chronische Wundpatienten als angenommen
}

Innovatives Wundmanagement hat positive Auswirkungen für die Betroffenen. Als Folge davon wertet die Initiative Wund?Gesund! die Ergebnisse einer kürzlich präsentierten Umfrage zur Wundversorgung in Österreich, die eine deutlich niedrigere Zahl an Betroffenen als bisher angenommen ergab. Aber das Verbesserungspotential ist weiter hoch: Noch immer werden die meisten Menschen mit chronischen Wunden mit einer traditionellen Wundversorgung behandelt. Und auch der Informationsbedarf der Patienten ist groß.

Bisher ging man von 400.000 Patienten mit chronischen Wunden in Österreich aus. Allerdings basierte diese Annahme auf einer Schätzung. Der Wundreport 2015, beauftragt von der Initiative Wund? Gesund! und durchgeführt von IFES, zeigt nun, dass die Zahl auf 250.000 nach unten korrigiert werden kann. Diese erste Großerhebung mit 7600 Befragten ergab eine Häufigkeit von fünf bis 5,8 Prozent Patienten mit chronischen Wunden. Dabei handelte es sich um eine reine Bevölkerungserhebung, die Spitäler und Pflegeheime nicht erfasste. Mit 402 Betroffenen und Angehörigen wurden dann in persönlichen Interviews die Themen Gründe und Entwicklungsverlauf der Wunde, Verbandwechsel, aktuelle Wundversorgung, Zusatzkosten und Selbstfinanzierung sowie Zufriedenheit mit der Krankenversicherung und Informationsbedarf eingehender erfragt. „Die Studie zeigt erstmals auf," so Dr. Gert Feistritzer von IFES, welche Auswirkungen chronische Wunden und deren traditionelle Versorgung auf die

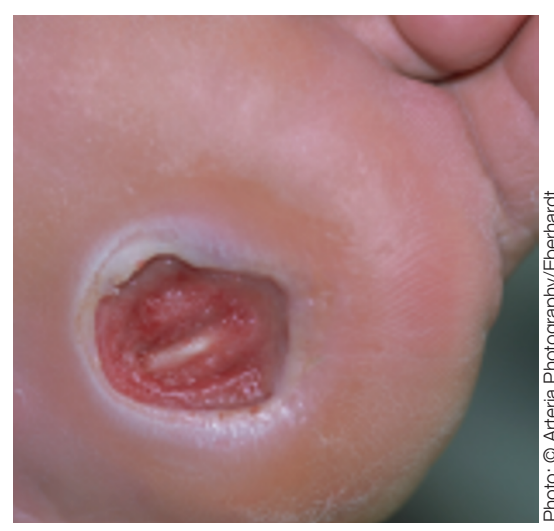

Betroffenen selbst, aber auch auf die Volkswirtschaft im Ganzen haben“. Die Studie ergab, dass nur 15 Prozent der Erkrankten mit modernen Wundprodukten behandelt werden, wobei dies überwiegend Personen mit höherem Einkommen sind. Auch wenn Personen mit geringerem Haushaltseinkommen logischerweise geringere Bereitschaft - und Möglichkeit angeben, selbst zu bezahlen, ist die Bereitschaft dazu insgesamt mit 56 Prozent doch relativ hoch. „Es gibt offenbar ein großes Informationsdefizit", stellt Feistritzer fest.

\section{Zu wenig Information über Kosten und Heilungsverlauf}

Das zeigt sich auch im Verhältnis zu den Krankenversicherungen, von denen sich die Patienten mehr Kostenrückerstattung und bessere Informationen wünschen. Denn auch bei der traditionellen Wundversorgung, mit der 80 Prozent der Befragten behandelt werden, sind derzeit fast zwei Drittel der Patienten mit Zusatzkosten konfrontiert. Patienten mit Zusatzversicherung sind bezüglich Kostenrückerstattung wesentlich zufriedener als jene mit der gesetzlichen Krankenkasse. Fast ein Drittel der Befragten ist auch mit der Informationsleistung ihrer gesetzlichen Krankenversicherung in Bezug auf die Wundversorgung nicht zufrieden.

Die überwiegend angewendete traditionelle Wundversorgung führt auch zu einer relativ hohen Verbandwechselfrequenz alle drei Tage bei knapp drei Viertel der Befragten, mehr als die Hälfte der Betroffenen kann den Verbandwechsel nicht selbst durchführen und muss daher einen niedergelassenen Arzt oder ein Krankenhaus aufsuchen, bei etwa einem Fünftel führen entweder die mobile Pflege, ein Angehöriger oder der Patient selbst den Verbandwechsel durch. 80 Prozent der chronischen Wunden werden durch Erkrankungen verursacht oder sind Folge einer Behandlung.

Mit den ärztlichen Informationen zum Heilungsverlauf der Wunde sind 42 Prozent der Befragten nicht zufrieden: Sie werden beim Verbandwechsel nicht ausreichend oder gar nicht über den Zustand der Wunde aufgeklärt und knapp zwei Drittel erleben den Verbandwechsel als schmerzhaft. 27 Prozent der Befragten erleben durch die Wunde starke Schmerzen. Einen der Vorteile der modernen Wundversorgung sehen die Befragten denn auch in einem geringeren Schmerzempfinden, weniger Geruchsbelästigung und einer positiveren Wundentwicklung.

\section{Kosten als Gesamtpaket}

Das Argument der höheren Kosten für die moderne Wundversorgung müsse im Gesamtzusammenhang betrachtet werden, fordert die Initiative Wund?Gesund!. Eine faire Preisgestaltung müsse auf der Basis von Gesamtbehandlungskosten und nicht auf Basis der Stückkosten betrachtet werden. „Unsere Produkte bieten einen Mehrwert", konstatierte Mag. Friedrich Thomasberger, Präsident der AustroMed, der Interessensvertretung der Medizinprodukte-Unternehmen und Geschäftsführer der Paul Hartmann GmbH: „Durch den Einsatz von innovativen und modernen Wundprodukten werden der Heilungsprozess beschleunigt, die Behandlungszeit verkürzt und somit Kosten eingespart." Die moderne Wundbehandlung belastet das System weniger und erhöht den Patientenkomfort durch weniger Schmerzen, weniger Geruch und schnellere Heilung. Das erhöhe zusätzlich die Compliance. Notwendig sei auch eine gezielte Aufklärung der Bevölkerung.

\section{Breite Beteiligung am Wundmanagement}

Im Krankenhaus Göttlicher Heiland wurde bereits vor einigen Jahren ein Integratives Wundmanagement etabliert, das alle Beteiligten zusammenbringt: Verschiedene Fachrichtungen, verschiedene Berufsgruppen, Patienten und Angehörige, „denn“, so Dr. Markus Duft, Vorstand des Vereins Wundmanagement Wien und Chirurg am KH Göttlicher Heiland in Wien, „wir müssen die Gesundheitskompetenz des Patienten schärfen." Und Duft unterstrich die große Bedeutung der Pflege: „Die meiste Arbeit macht die Pflege, nicht die Medizin!“ $k i$

Quelle: Präsentation Wundreport 2015 der Initiative Wund?Gesund!, 1. Oktober 2015, Wien 